\title{
Extractable aluminium, iron and manganese in mineral soils II Extractability by oxalate and pyrophosphate
}

\author{
RAINA NISKANEN \\ Department of Agricultural Chemistry, University of Helsinki, \\ SF-00710 Helsinki, Finland
}

\begin{abstract}
The extractability of aluminium, iron and manganese by $0.05 \mathrm{M}$ oxalate and pyrophosphate was studied in samples of 23 mineral soils. Dilute extractants were studied because conventional reagents may cause problems in analytical work. The mean values for $\mathrm{Al}$, Fe and Mn extracted by conventional Tamm's oxalate were 67,81 and $1.5 \mathrm{mmol} / \mathrm{kg}$ soil, respectively. On the average, $0.05 \mathrm{M}$ oxalate solutions at $\mathrm{pH} 2.9$ and 4.2 extracted $\mathrm{Al}, \mathrm{Fe}$ and $\mathrm{Mn}$ amounts that were 103, 113 and $87 \%$ and 72,82 and $83 \%$ of the amounts extractable by Tamm's oxalate, respectively. Eeach metal released by $0.05 \mathrm{M}$ oxalates correlated closely with that dissolved by Tamm's oxalate; the $\mathrm{r}$ values ranged from $0.967^{* * *}$ to $0.997^{* * *}$. The mean values for $\mathrm{Al}, \mathrm{Fe}$ and $\mathrm{Mn}$ extracted by $0.1 \mathrm{M} \mathrm{Na}_{4} \mathrm{P}_{2} \mathrm{O}_{7}$ and $0.05 \mathrm{M} \mathrm{K}_{4} \mathrm{P}_{2} \mathrm{O}_{7}$ were 38, 28 and 0.6 and 33,29 and $0.6 \mathrm{mmol} / \mathrm{kg}$ soil, respectively. The amount of each metal extracted by $\mathrm{Na}_{4} \mathrm{P}_{2} \mathrm{O}$, correlated closely with that released by $\mathrm{K}_{4} \mathrm{P}_{2} \mathrm{O}_{7}$; the $\mathrm{r}$ values ranged from $0.87^{* * *}$ to $0.97^{* * *}$,
\end{abstract}

Index words: amorphous oxides, complexing agents, soil organic carbon, soil extraction

\section{Introduction}

Acid ammonium oxalate (TАMм 1922, Schwertmann 1964, McKeague and Day 1966) and $0.1 \mathrm{M} \mathrm{Na} \mathrm{P}_{2} \mathrm{O}_{7}$ (Aleksandrova 1960, McKeague 1967) are conventionally used for the extraction of aluminium, iron and manganese from soil. Amorphous oxides and metals bound to organic matter in soil are dissolved by these solutions. The use of these reagents, however, may cause some problems in analytical work.
Traditional colorimetric, titrimetric and gravimetric techniques have commonly been superseded by atomic absorption spectrophotometry. Application of this technique may sometimes be hampered by the tendency of the nebulizer and burner slot to clog when solutions of high salt concentration are aspirated. Acid ammonium oxalate has often proved to be especially troublesome in this respect (e.g. Webier et al. 1974, Searly and 
Daly 1977, Simmons and Plues-Foster 1977). Difficulties may partially be overcome by dilution of the solutions to be analyzed. Pyrophosphate is difficult to use as an extractant because of soil dispersion. Especially with clay soils, normal filtration through paper is not enough for clarifying extracts (SHELDRICK and McKeague 1975, Schuppli et al. 1983).

These difficulties in analytical work should be alleviated by the use of more dilute extractants. A previous paper (NISKANEN 1989) showed that the ability of oxalate and pyrophosphate to extract soil aluminium, iron and manganese depends on the $\mathrm{pH}$ value, and that the extractability by $0.05 \mathrm{M}$ reagents can reach the same order of magnitude as that by conventional methods. The aim of this paper was to study the possibility of employing $0.05 \mathrm{M}$ oxalate and pyrophosphate solutions instead of the traditional reagents for the extraction of aluminium, iron and manganese from soil.

\section{Material and methods}

The material was collected at 14 sampling sites on the Viikki Experimental Farm, University of Helsinki (Nos. 1-9) and in South Karelia (Imatra) (Nos. 10-14) (Table 1). At sampling sites Nos. $1-9$ both surface $(0-20 \mathrm{~cm})$ and deeper layer $(20-40 \mathrm{~cm})$ samples were taken; at sampling sites Nos. 10-14 only surface samples were taken.

The soils were air-dried and ground to pass through a $2-\mathrm{mm}$ sieve. The particle-size distribution of the inorganic material in the soil was determined by the pipette method (ElONEN 1971), the organic carbon content by the ALTEN wet combustion method (GRAHAM 1948). The soil pH was measured in a soil-0.01 M $\mathrm{CaCl}_{2}$ suspension (1:2.5 v/v) (RYTI 1965).

The soils were extracted by the methods given in Table 2. $\mathrm{Na}_{4} \mathrm{P}_{2} \mathrm{O}_{7}$ extracts were clarified by the addition of $1 \mathrm{M} \mathrm{HCl}$ and then filtration through hard paper, $\mathrm{K}_{4} \mathrm{P}_{2} \mathrm{O}_{7}$ ex-

Table 1. Soil samples $(\mathrm{a}=0-20 \mathrm{~cm}, \mathrm{~b}=20-40 \mathrm{~cm}, \mathrm{~V}=$ virgin soil).

\begin{tabular}{|c|c|c|c|c|c|c|c|}
\hline \multirow{2}{*}{$\begin{array}{l}\text { Soil } \\
\text { No. }\end{array}$} & \multirow[t]{2}{*}{$\mathrm{pH}\left(\mathrm{CaCl}_{2}\right)$} & \multirow{2}{*}{$\underset{\%}{\text { Organic } C}$} & \multicolumn{5}{|c|}{ Particle-size distribution $(\mu \mathrm{m}), \%$} \\
\hline & & & $<2$ & $2-20$ & $20-60$ & $60-200$ & $>200$ \\
\hline 1a & 4.6 & 3.3 & 37 & 13 & 15 & 30 & 4 \\
\hline $\mathrm{Ib}$ & 5.2 & 0.8 & 39 & 6 & 14 & 38 & 3 \\
\hline $2 \mathrm{a}$ & 4.3 & 4.7 & 36 & 8 & 21 & 34 & 3 \\
\hline $2 b$ & 5.0 & 1.0 & 26 & 2 & 23 & 42 & 6 \\
\hline $3 \mathrm{a}$ & 5.9 & 5.3 & 58 & 14 & 12 & 13 & 3 \\
\hline $3 \mathrm{~b}$ & 5.6 & 1.7 & 45 & 19 & 16 & 17 & 3 \\
\hline $4 \mathrm{a} \mathrm{V}$ & 3.5 & 9.2 & 3 & 7 & 11 & 29 & 50 \\
\hline $4 \mathrm{~b} \mathrm{~V}$ & 4.1 & 2.7 & 2 & 5 & 10 & 28 & 55 \\
\hline $5 \mathrm{a} \mathrm{V}$ & 4.6 & 1.5 & 4 & 16 & 30 & 25 & 26 \\
\hline $5 \mathrm{~b} \mathrm{~V}$ & 4.3 & 2.0 & 11 & 29 & 41 & 12 & 7 \\
\hline $6 a$ & 4.8 & 2.4 & 29 & 30 & 20 & 15 & 6 \\
\hline $6 \mathrm{~b}$ & 5.2 & 1.4 & 49 & 20 & 11 & 16 & 4 \\
\hline $7 a$ & 4.5 & 3.4 & 43 & 33 & 18 & 3 & 2 \\
\hline $7 b$ & 4.8 & 2.6 & 47 & 30 & 18 & 5 & 0 \\
\hline $8 a$ & 5.3 & 4.4 & 10 & 7 & 15 & 61 & 7 \\
\hline $8 \mathrm{~b}$ & 5.3 & 3.0 & 11 & 7 & 17 & 59 & 6 \\
\hline $9 \mathrm{a} \mathrm{V}$ & 4.4 & 3.0 & 2 & 2 & 3 & 15 & 77 \\
\hline $9 \mathrm{~b} \mathrm{~V}$ & 4.6 & 0.8 & 2 & 1 & 7 & 35 & 56 \\
\hline $10 \mathrm{a} \mathrm{V}$ & 4.3 & 6.0 & 5 & 6 & 5 & 22 & 62 \\
\hline $11 \mathrm{a}$ & 5.7 & 3.8 & 22 & 31 & 27 & 16 & 5 \\
\hline $12 \mathrm{a}$ & 5.1 & 3.6 & 13 & 20 & 27 & 31 & 9 \\
\hline $13 \mathrm{a}$ & 4.7 & 4.7 & 51 & 27 & 11 & 5 & 7 \\
\hline $14 a$ & 5.2 & 3.2 & 31 & 43 & 13 & 7 & 5 \\
\hline$\overline{\mathrm{x}}$ & 4.8 & 3.2 & 25 & 16 & 17 & 24 & 18 \\
\hline s & 0.6 & 1.9 & 19 & 12 & 9 & 16 & 24 \\
\hline range & $3.5-5.9$ & $0.8-9.2$ & $2-58$ & $1-43$ & $3-41$ & $3-61$ & $0-77$ \\
\hline
\end{tabular}


Table 2. Extraction methods.

\begin{tabular}{lcccc}
\hline Extractant & $\mathrm{pH}$ & $\begin{array}{c}\text { Extraction } \\
\text { ratio, w/v }\end{array}$ & $\begin{array}{l}\text { Shaking } \\
\text { time, } \mathrm{h}\end{array}$ & Reference \\
\hline 1. $0.18 \mathrm{M}$ ammonium oxalate, $0.10 \mathrm{M}$ oxalic acid & 3.3 & $1: 20$ & 2 & TAMM 1922 \\
2. $0.026 \mathrm{M}$ ammonium oxalate, $0.024 \mathrm{M}$ oxalic acid & 2.9 & $1: 20$ & 2 & \\
3. $0.041 \mathrm{M}$ ammonium oxalate, $0.009 \mathrm{M}$ oxalic acid & 4.2 & $1: 200$ & 3 & \\
4. $0.029 \mathrm{M} \mathrm{ammonium} \mathrm{oxalate,} 0.021 \mathrm{M}$ oxalic acid & 3.3 & $1: 20$ & 2 & \\
5. $0.1 \mathrm{M} \mathrm{Na}_{4} \mathrm{P}_{2} \mathrm{O}_{7}$ & 10 & $1: 20$ & 4 & MCKEAGUE 1967 \\
6. $0.05 \mathrm{M} \mathrm{K}_{4} \mathrm{P}_{2} \mathrm{O}_{7}$ & 10 & $1: 100$ & 3 & \\
\hline
\end{tabular}

tracts by filtration through $0.2-\mu \mathrm{m}$ membrane filters. Aluminium, iron and manganese in filtrates were determined by atomic absorption spectrophotometry, iron and manganese with air-acetylene and aluminium with $\mathrm{N}_{2} \mathrm{O}$ acetylene flame. The experiment was carried out in duplicate.

\section{Results and discussion}

\section{Extractability by oxalate}

Aluminium and iron extracted by.Tamm's oxalate, respectively, were nearly of the same order of magnitude as those extracted by 0.05

Table 3. Extractable aluminium, $\mathrm{mmol} / \mathrm{kg}$ soil.*

\begin{tabular}{|c|c|c|c|c|c|}
\hline \multirow{2}{*}{$\begin{array}{l}\text { Soil } \\
\text { No. }\end{array}$} & \multicolumn{2}{|l|}{$p+3.3$} & \multicolumn{3}{|l|}{ Al extracted by } \\
\hline & $\begin{array}{l}\text { Tamm's } \\
\text { oxalate }\end{array}$ & $\begin{array}{c}0.05 \mathrm{M} \text { oxalate } \\
\mathrm{pH} 2.9\end{array}$ & $\begin{array}{c}0.05 \mathrm{M} \text { oxalate } \\
\mathrm{pH} 4.2\end{array}$ & $\begin{array}{c}0.1 \mathrm{M} \\
\mathrm{Na}_{4} \mathrm{P}_{2} \mathrm{O}_{7}\end{array}$ & $\begin{array}{l}0.05 \mathrm{M} \\
\mathrm{K}_{4} \mathrm{P}_{2} \mathrm{O}_{7}\end{array}$ \\
\hline $1 a$ & $31^{\mathrm{d}}$ & $34^{d}$ & $17{ }^{c}$ & $14^{b}$ & $10^{a}$ \\
\hline $1 \mathrm{~b}$ & $17^{b}$ & $29^{c}$ & $8^{a}$ & $2^{a}$ & $4^{a}$ \\
\hline $2 \mathrm{a}$ & $49^{d}$ & $51^{\mathrm{d}}$ & $38^{c}$ & $28^{\mathrm{b}}$ & $23^{a}$ \\
\hline $2 b$ & $11^{b}$ & $12^{b}$ & $5^{a}$ & $4^{a}$ & $4^{a}$ \\
\hline $3 a$ & $104^{e}$ & $92^{\mathrm{d}}$ & 70 & $52^{\mathrm{b}}$ & $44^{a}$ \\
\hline $3 \mathrm{~b}$ & $63^{c}$ & $68^{\mathrm{d}}$ & $27^{a}$ & $30^{b}$ & $26^{a}$ \\
\hline $4 a$ & $55^{\mathrm{c}}$ & $51^{\mathrm{bc}}$ & $41^{\text {a }}$ & $47^{a b}$ & $41^{a}$ \\
\hline $4 b$ & $81^{b}$ & $82^{\mathrm{b}}$ & $58^{a}$ & $81^{b}$ & $79^{b}$ \\
\hline $5 \mathrm{a}$ & $34^{b}$ & $35^{b}$ & $23^{a}$ & $23^{a}$ & $22^{a}$ \\
\hline $5 b$ & $58 \mathrm{c}$ & $61^{\mathrm{c}}$ & $40^{a}$ & $46^{b}$ & $44^{a b}$ \\
\hline $6 a$ & $53^{d}$ & $54^{d}$ & $37^{c}$ & $28^{\mathrm{b}}$ & $21^{\mathrm{a}}$ \\
\hline $6 \mathrm{~b}$ & $52^{c}$ & 54 & $34^{b}$ & $15^{\mathrm{a}}$ & $11^{a}$ \\
\hline $7 \mathrm{a}$ & $79^{d}$ & $74^{d}$ & $66^{c}$ & $37^{b}$ & $31^{a}$ \\
\hline $7 b$ & $76^{d}$ & $68^{c}$ & 64 & $23^{b}$ & $16^{\mathrm{a}}$ \\
\hline $8 \mathrm{a}$ & $23^{c}$ & $24^{\mathrm{d}}$ & $19^{b}$ & $15^{\mathrm{a}}$ & $15^{a}$ \\
\hline $8 \mathrm{~b}$ & $21^{\mathrm{c}}$ & $23^{c}$ & $15^{b}$ & $10^{a}$ & $10^{a}$ \\
\hline $9 \mathrm{a}$ & $59^{b}$ & 69 & $54^{b}$ & $46^{a}$ & $46^{a}$ \\
\hline $9 b$ & $104^{b}$ & $92^{\mathrm{b}}$ & $110^{b}$ & $54^{\mathrm{a}}$ & $56^{\mathrm{a}}$ \\
\hline $10 \mathrm{a}$ & $67^{d}$ & $65^{\mathrm{d}}$ & $57 c$ & $46^{b}$ & $35^{a}$ \\
\hline $11 \mathrm{a}$ & $80^{d}$ & $76^{d}$ & $64^{c}$ & $42^{b}$ & $29^{a}$ \\
\hline $12 \mathrm{a}$ & $186^{c}$ & $161^{\mathrm{b}}$ & $158^{\mathrm{b}}$ & $92^{a}$ & $82^{a}$ \\
\hline $13 a$ & $159^{\mathrm{d}}$ & 134 & $115^{b c}$ & $108^{b}$ & $74^{a}$ \\
\hline $14 \mathrm{a}$ & $78^{c}$ & $73^{d}$ & $53^{c}$ & $40^{b}$ & $30^{2}$ \\
\hline $\bar{x}$ & 67 & 64 & 51 & 38 & 33 \\
\hline $\mathbf{s}$ & 42 & 34 & 37 & 27 & 23 \\
\hline range & $11-186$ & $12-161$ & $5-158$ & $2-108$ & $4-82$ \\
\hline
\end{tabular}

* Each soil tested separately. Values marked with the same letter do not deviate, with $5 \%$ risk. 


\begin{tabular}{|c|c|c|c|c|c|}
\hline \multirow{2}{*}{$\begin{array}{l}\text { Soil } \\
\text { No. }\end{array}$} & \multicolumn{5}{|c|}{ Fe extracted by } \\
\hline & $\begin{array}{l}\text { Tamm's } \\
\text { oxalate }\end{array}$ & $\begin{array}{c}0.05 \mathrm{M} \text { oxalate } \\
\mathrm{pH} 2.9\end{array}$ & $\begin{array}{c}0.05 \mathrm{M} \text { oxalate } \\
\mathrm{pH} 4.2\end{array}$ & $\begin{array}{c}0.1 \mathrm{M} \\
\mathrm{Na}_{4} \mathrm{P}_{2} \mathrm{O}_{7}\end{array}$ & $\begin{array}{l}0.05 \mathrm{M} \\
\mathrm{K}_{4} \mathrm{P}_{2} \mathrm{O}_{7}\end{array}$ \\
\hline la & $34^{c}$ & $43^{d}$ & $29^{b}$ & $13^{a}$ & $12^{a}$ \\
\hline $1 b$ & $28^{c}$ & $37^{d}$ & $20^{b}$ & $5^{a}$ & $5^{a}$ \\
\hline $2 \mathrm{a}$ & $47^{\mathrm{b}}$ & $59^{\mathrm{d}}$ & $55^{\mathrm{c}}$ & $26^{a}$ & $28^{a}$ \\
\hline $2 b$ & $11^{\mathrm{bc}}$ & $14^{c}$ & $9^{b}$ & $3^{a}$ & $5^{\mathrm{a}}$ \\
\hline $3 \mathrm{a}$ & $107^{c}$ & $120^{d}$ & $97^{\mathrm{b}}$ & $42^{a}$ & $41^{a}$ \\
\hline $3 b$ & $157^{c}$ & $162^{c}$ & $112^{b}$ & $40^{\mathrm{a}}$ & $41^{\mathrm{a}}$ \\
\hline $4 a$ & $49^{\text {dd }}$ & $56^{\mathrm{d}}$ & $41^{b}$ & $31^{a}$ & $47^{\mathrm{bc}}$ \\
\hline $4 b$ & $44^{c}$ & $55^{\mathrm{d}}$ & $35^{b}$ & $27^{a}$ & $36^{\mathrm{b}}$ \\
\hline $5 \mathrm{a}$ & $23^{\mathrm{c}}$ & $31^{\mathrm{d}}$ & $19^{b}$ & $11^{\mathrm{a}}$ & $12^{a}$ \\
\hline $5 b$ & $60^{d}$ & $71^{e}$ & $55^{c}$ & $34^{a}$ & $40^{b}$ \\
\hline $6 a$ & $79^{\circ}$ & $95^{\mathrm{d}}$ & $68^{b}$ & $27^{a}$ & $30^{2}$ \\
\hline $6 b$ & $93^{d}$ & $105^{e}$ & $70^{\circ}$ & $20^{b}$ & $17^{a}$ \\
\hline $7 a$ & $211^{\mathrm{d}}$ & $192^{c}$ & $174^{b}$ & $42^{a}$ & $50^{\mathrm{a}}$ \\
\hline $7 b$ & $223^{c}$ & $202^{\mathrm{b}}$ & $213^{c}$ & $38^{a}$ & $40^{a}$ \\
\hline $8 \mathrm{a}$ & $140^{\circ}$ & $144^{c}$ & $121^{\mathrm{b}}$ & $69^{a}$ & $78^{a}$ \\
\hline $8 \mathrm{~b}$ & $115^{b}$ & $135^{c}$ & $101^{b}$ & $47^{a}$ & $53^{a}$ \\
\hline $9 a$ & $30^{\circ}$ & $42^{\mathrm{d}}$ & $25^{\mathrm{b}}$ & $13^{a}$ & $15^{\mathrm{a}}$ \\
\hline $9 b$ & $32^{b}$ & $39^{\circ}$ & $31^{\mathrm{b}}$ & $10^{\mathrm{a}}$ & $9^{a}$ \\
\hline $10 a$ & $34^{c}$ & $37^{c}$ & $23^{b}$ & $18^{a}$ & $17^{\mathrm{a}}$ \\
\hline $11 \mathrm{a}$ & $60^{d}$ & $61^{d}$ & 37 & $16^{b}$ & $12^{a}$ \\
\hline $12 \mathrm{a}$ & $53^{\mathrm{d}}$ & $52^{\mathrm{d}}$ & $36^{c}$ & $24^{b}$ & $20^{2}$ \\
\hline $13 a$ & $159^{e}$ & $124^{d}$ & $113^{c}$ & $55^{\text {b }}$ & $40^{\mathrm{a}}$ \\
\hline $14 a$ & $84^{d}$ & $86^{d}$ & $59^{\circ}$ & $23^{b}$ & $18^{\mathrm{a}}$ \\
\hline$\overline{\mathrm{x}}$ & 81 & 85 & 67 & 28 & 29 \\
\hline $\mathbf{s}$ & 60 & 53 & 52 & 17 & 19 \\
\hline range & $11-223$ & $14-202$ & $9-213$ & $3-69$ & $5-78$ \\
\hline
\end{tabular}

* Each soil tested separately. Values marked with the same letter do not deviate, with $5 \%$ risk.

$\mathrm{M}$ oxalate at $\mathrm{pH} 2.9$, but higher than those extracted by $0.05 \mathrm{M}$ oxalate at $\mathrm{pH} 4.2$ (Tables 3 and 4). In most samples Tamm's oxalate extracted more manganese than that extracted by dilute oxalates (Table 5). The metals extracted by dilute oxalates correlated closely to those extracted by Tamm's oxalate. Regression equations describing the relationship between extractabilities and corresponding linear correlation coefficients were as follows: that extractable by Tamm's oxalate (Table 6). The relative extractability of aluminium was lower than that of iron and manganese, being at the lowest $43 \%$ of the Tamm's oxalateextractable aluminium (Table 6). In order for the extractability by $0.05 \mathrm{M}$ oxalate to reach the same order of magnitude as that by Tamm's oxalate, the $\mathrm{pH}$ of the extractant must be lower than 4.2 .

The lower extractability by oxalate at $\mathrm{pH}$

$\mathrm{Al}(\mathrm{Tamm})(\mathrm{mmol} / \mathrm{kg})=-11.41+1.22 \mathrm{Al}($ oxal. $\mathrm{pH} 2.9)(\mathrm{mmol} / \mathrm{kg})$

$$
\begin{aligned}
& \mathrm{r}=0.992^{* * *} \\
& \mathrm{r}=0.967^{* * *} \\
& \mathrm{r}=0.982^{* * *} \\
& \mathrm{r}=0.981^{* * *} \\
& \mathrm{r}=0.984 * * * \\
& \mathrm{r}=0.997 * * *
\end{aligned}
$$$$
\mathrm{Al}(\mathrm{Tamm})(\mathrm{mmol} / \mathrm{kg})=10.32+1.11 \mathrm{Al}(\text { oxal. } \mathrm{pH} 4.2)(\mathrm{mmol} / \mathrm{kg})
$$$$
\mathrm{Fe}(\mathrm{Tamm})(\mathrm{mmol} / \mathrm{kg})=-13.14+1.11 \mathrm{Fe}(\text { oxal. } \mathrm{pH} 2.9)(\mathrm{mmol} / \mathrm{kg})
$$$$
\mathrm{Mn}(\mathrm{Tamm})(\mu \mathrm{mol} / \mathrm{kg})=55.98+1.10 \mathrm{Mn}(\text { oxal. } \mathrm{pH} 2.9)(\mu \mathrm{mol} / \mathrm{kg})
$$$$
\mathrm{Mn}(\mathrm{Tamm})(\mu \mathrm{mol} / \mathrm{kg})=83.99+1.05 \mathrm{Mn}(\mathrm{oxal} . \mathrm{pH} 4.2)(\mu \mathrm{mol} / \mathrm{kg})
$$

The extractability of metals by $0.05 \mathrm{M}$ oxalate at $\mathrm{pH} 4.2$ was commonly $<100 \%$ of
4.2 is in agreement with the results of McKeague and Day (1966). They found that 


\begin{tabular}{|c|c|c|c|c|c|}
\hline \multirow{2}{*}{$\begin{array}{l}\text { Soil } \\
\text { No. }\end{array}$} & \multicolumn{5}{|c|}{ Mn extracted by } \\
\hline & $\begin{array}{l}\text { Tamm's } \\
\text { oxalate }\end{array}$ & $\begin{array}{c}0.05 \text { M oxalate } \\
\text { pH } 2.9\end{array}$ & $\begin{array}{c}0.05 \mathrm{M} \text { oxalate } \\
\mathrm{pH} 4.2\end{array}$ & $\begin{array}{c}0.1 \mathrm{M} \\
\mathrm{Na}_{4} \mathrm{P}_{2} \mathrm{O}_{7}\end{array}$ & $\begin{array}{l}0.05 \mathrm{M} \\
\mathrm{K}_{4} \mathrm{P}_{2} \mathrm{O}_{7}\end{array}$ \\
\hline $1 \mathrm{a}$ & $228^{a}$ & $228^{a}$ & $260^{\mathrm{a}}$ & $118^{\mathrm{a}}$ & $273^{a}$ \\
\hline lb & $410^{\circ}$ & $408^{\mathrm{c}}$ & $346^{\mathrm{bc}}$ & $155^{a}$ & $304^{b}$ \\
\hline $2 \mathrm{a}$ & $419^{d}$ & $355^{\mathrm{bc}}$ & $346^{b}$ & $209^{a}$ & $364^{c}$ \\
\hline $2 b$ & $346^{c}$ & $346^{c}$ & $260^{b}$ & $151^{\mathrm{a}}$ & $315^{c}$ \\
\hline $3 \mathrm{a}$ & $1092^{\mathrm{d}}$ & $850^{\circ}$ & $1214^{e}$ & $233^{a}$ & $364^{b}$ \\
\hline $3 b$ & $160^{\mathrm{sd}}$ & $191^{d}$ & $87^{b}$ & $36^{a}$ & $122^{\mathrm{bc}}$ \\
\hline $4 a$ & $1138^{c}$ & $869^{b}$ & $868^{b}$ & $596^{\mathrm{a}}$ & $1011^{\mathrm{bc}}$ \\
\hline $4 b$ & $3640^{\circ}$ & $3986^{c}$ & $3559^{\mathrm{c}}$ & $837^{\mathrm{a}}$ & $1661^{b}$ \\
\hline $5 \mathrm{a}$ & $301^{\mathrm{c}}$ & $282^{b c}$ & $173^{a}$ & $137^{a}$ & $242^{\mathrm{b}}$ \\
\hline $5 b$ & $956^{d}$ & $784^{c}$ & $824^{c}$ & $346^{a}$ & $513^{b}$ \\
\hline $6 a$ & $3367^{d}$ & $3367^{d}$ & $3164^{c}$ & $482^{a}$ & $728^{b}$ \\
\hline $6 b$ & $1911^{\mathrm{d}}$ & $1531^{\mathrm{b}}$ & $1734^{c}$ & $164^{\mathrm{a}}$ & $242^{a}$ \\
\hline $7 \mathrm{a}$ & $410^{c}$ & $346^{\mathrm{bc}}$ & $303^{b c}$ & $69^{a}$ & $242^{b}$ \\
\hline $7 b$ & $273^{c}$ & $164^{b}$ & $173^{b}$ & $87^{a}$ & $152^{b}$ \\
\hline $8 \mathrm{a}$ & $501^{\mathrm{c}}$ & $382^{b}$ & $433^{b c}$ & $190^{a}$ & $274^{a}$ \\
\hline $8 b$ & $437^{e}$ & $337^{d}$ & $260^{\circ}$ & $82^{a}$ & $137^{\mathrm{b}}$ \\
\hline $9 \mathrm{a}$ & $1211^{\mathrm{c}}$ & $1008^{b c}$ & $910^{b c}$ & $301^{a}$ & $637^{a b}$ \\
\hline $9 b$ & $528^{e}$ & $497^{d}$ & $433^{c}$ & $100^{\mathrm{a}}$ & $137^{b}$ \\
\hline $10 \mathrm{a}$ & $1529^{a}$ & $1222^{a}$ & $1300^{\mathrm{a}}$ & $1984^{b}$ & $1255^{a}$ \\
\hline $11 \mathrm{a}$ & $4004^{d}$ & $3640^{\circ}$ & $3553^{\mathrm{c}}$ & $1766^{b}$ & $1183^{a}$ \\
\hline $12 \mathrm{a}$ & $2212^{b}$ & $2184^{b}$ & $2254^{b}$ & $2276^{\mathrm{b}}$ & $1664^{a}$ \\
\hline $13 \mathrm{a}$ & $2821^{\mathrm{e}}$ & $1671^{\mathrm{e}}$ & $2340^{d}$ & $1183^{b}$ & $698^{a}$ \\
\hline $14 a$ & $7490^{\circ}$ & $6243^{b}$ & $7055^{c}$ & $1693^{a}$ & $1365^{a}$ \\
\hline$\overline{\mathrm{x}}$ & 1538 & 1343 & 1385 & 574 & 604 \\
\hline s & 1753 & 1562 & 1664 & 697 & 502 \\
\hline range & $160-7490$ & $164-6243$ & $87-7055$ & $36-2276$ & $122-1664$ \\
\hline
\end{tabular}

* Each soil tested separately. Values marked with the same letter do not deviate, with $5 \%$ risk.

approximately equal amounts of iron and aluminium, respectively, were extracted by oxalate at $\mathrm{pH} 2$ and 3 , but that solution of $\mathrm{pH}$ 4.2 extracted somewhat less metals. The solutions of $\mathrm{pH} 2$ and 3 also maintained their initial $\mathrm{pH}$, whereas the solution of $\mathrm{pH} 4.2$ was not buffered strongly enough to maintain a constant $\mathrm{pH}$ in extracts of soils rich in iron and aluminium.

The $\mathrm{pH}$ of oxalate was lowered to 2.9 because a preliminary experiment showed that the extractability of iron by $0.05 \mathrm{M}$ oxalate at $\mathrm{pH} 3.3$ was lower than that extractable by Tamm's oxalate (Table 7). In most soils, the extractability of aluminium and iron by oxalate at $\mathrm{pH} 2.9$, unlike that of manganese, was not lower than the extractability by Tamm's solution (Table 6). The extractability of aluminium was exceptionally high in soil
$1 \mathrm{~b}$, poor in aluminium.

Oxalate-extractable metals were not related to soil $\mathrm{pH}$ or organic carbon content. Only oxalate-extractable iron correlated weakly with the soil clay content. The linear correlation coefficients for the relationship between clay content and iron extracted by Tamm's solution and by oxalates of $\mathrm{pH} 2.9$ and 4.2 were $0.55^{* *}, 0.53^{*}$ and $0.52^{*}$, respectively.

\section{Extractability by pyrophosphate}

In most of the soils, the values of aluminium and iron, respectively, did not deviate statistically in both pyrophosphate extractions (Tables 3 and 4), whereas the manganese values deviated in most soils (Table 5). The metals released in two pyrophosphate extractions correlated closely, the regression equa- 


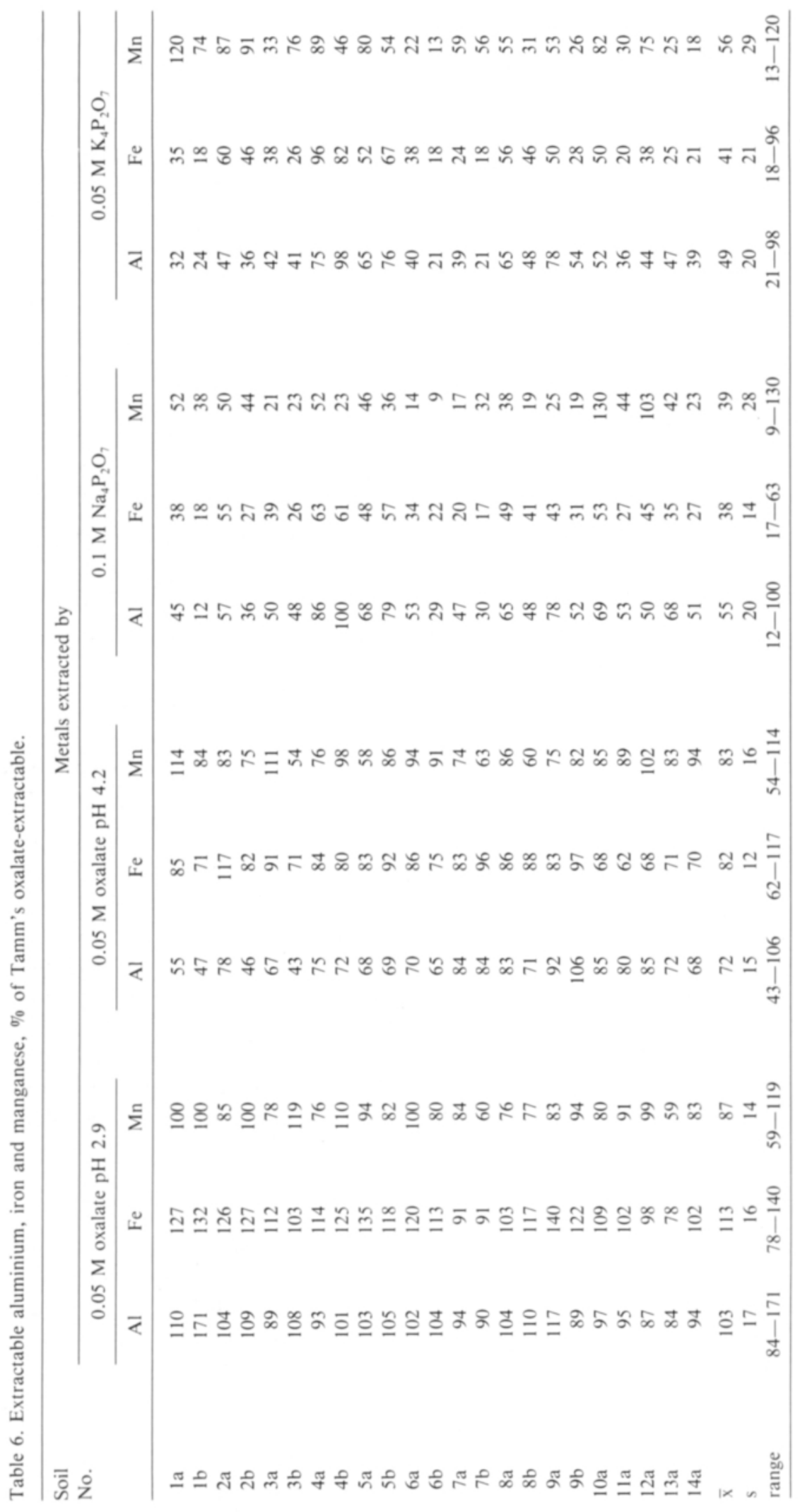


Table 7. Aluminium and iron extracted by $0.05 \mathrm{M}$ oxalate $\mathrm{pH} 3.3, \%$ of Tamm's oxalate-extractable.

\begin{tabular}{crc}
\hline Soil No. & Al & Fe \\
\hline la & 94 & 88 \\
1b & 135 & 84 \\
$2 \mathrm{~b}$ & 99 & 74 \\
3b & 101 & 75 \\
$12 \mathrm{a}$ & 68 & 69 \\
\hline
\end{tabular}

tions and corresponding linear correlation coefficients being as follows: aluminium were $0.49^{*}, 0.54^{* *}$ and $0.47^{*}$, respectively.

In pyrophosphate extracts of clay soils, metals are not exclusively in a dissolved form, but are also present in suspended material which is difficult to remove. This material may be fine particulate amorphous material, or it may have been formed by coagulation of solutes in the pyrophosphate extracts (Bascomb 1968, McKeague and Schuppli 1982). The methods used for clarifying of pyrophosphate extracts are high-speed cen-
$\mathrm{Al}\left(\mathrm{Na}_{4} \mathrm{P}_{2} \mathrm{O}_{7}\right)(\mathrm{mmol} / \mathrm{kg})=0.99+1.14 \mathrm{Al}\left(\mathrm{K}_{4} \mathrm{P}_{2} \mathrm{O}_{7}\right)(\mathrm{mmol} / \mathrm{kg})$

$\mathrm{Fe}\left(\mathrm{Na}_{4} \mathrm{P}_{2} \mathrm{O}_{7}\right)(\mathrm{mmol} / \mathrm{kg})=3.14+0.84 \mathrm{Fe}\left(\mathrm{K}_{4} \mathrm{P}_{2} \mathrm{O}_{7}\right)(\mathrm{mmol} / \mathrm{kg})$

$\mathrm{Mn}\left(\mathrm{Na}_{4} \mathrm{P}_{2} \mathrm{O}_{7}\right)(\mu \mathrm{mol} / \mathrm{kg})=-157+1.21 \mathrm{Mn}\left(\mathrm{K}_{4} \mathrm{P}_{2} \mathrm{O}_{7}\right)(\mu \mathrm{mol} / \mathrm{kg})$

$$
\begin{aligned}
& \mathrm{r}=0.968^{* * *} \\
& \mathrm{r}=0.945^{* * *} \\
& \mathrm{r}=0.872 * * *
\end{aligned}
$$

Pyrophosphate-extractable metals did not correlate statistically significantly with soil $\mathrm{pH}$ or clay content. In contrast, the pyrophosphate-extractable metals correlated with the oxalate-extractable metals. Regression equations describing the relationship between metals extracted by Tamm's oxalate and pyrophosphates and corresponding linear correlation coefficients were as follows: trifugation (McKeAgue 1967) and the addition of Superfloc, a flocculating agent, followed by centrifugation at low speed (SHELDRICK and McKeague 1975). The study of Schuppli et al. (1983) shows that centrifugation at high speed or at low speed with Superfloc is inadequate to sediment suspended material completely. Adding a salt, such as $\mathrm{Na}_{2} \mathrm{SO}_{4}$, is not effective at low concentration, and at high

$$
\begin{aligned}
& \mathrm{Al}(\mathrm{Tamm})(\mathrm{mmol} / \mathrm{kg})=13.37+1.40 \mathrm{Al}\left(\mathrm{Na}_{4} \mathrm{P}_{2} \mathrm{O}_{7}\right)(\mathrm{mmol} / \mathrm{kg}) \\
& \mathrm{Al}(\mathrm{Tamm})(\mathrm{mmol} / \mathrm{kg})=16.31+1.55 \mathrm{Al}\left(\mathrm{K}_{4} \mathrm{P}_{2} \mathrm{O}_{7}\right)(\mathrm{mmol} / \mathrm{kg}) \\
& \mathrm{Fe}(\mathrm{Tamm})(\mathrm{mmol} / \mathrm{kg})=5.39+2.76 \mathrm{Fe}\left(\mathrm{Na}_{4} \mathrm{P}_{2} \mathrm{O}_{7}\right)(\mathrm{mmol} / \mathrm{kg}) \\
& \mathrm{Fe}(\mathrm{Tamm})(\mathrm{mmol} / \mathrm{kg})=18.24+2.18 \mathrm{Fe}\left(\mathrm{K}_{4} \mathrm{P}_{2} \mathrm{O}_{7}\right)(\mathrm{mmol} / \mathrm{kg}) \\
& \mathrm{Mn}(\mathrm{Tamm})(\mu \mathrm{mol} / \mathrm{kg})=574+1.68 \mathrm{Mn}\left(\mathrm{Na}_{4} \mathrm{P}_{2} \mathrm{O}_{7}\right)(\mu \mathrm{mol} / \mathrm{kg}) \\
& \mathrm{Mn}(\mathrm{Tamm})(\mu \mathrm{mol} / \mathrm{kg})=27.97+2.50 \mathrm{Mn}\left(\mathrm{K}_{4} \mathrm{P}_{2} \mathrm{O}_{7}\right)(\mu \mathrm{mol} / \mathrm{kg})
\end{aligned}
$$

$$
\begin{aligned}
& r=0.892^{* * *} \\
& \mathrm{r}=0.837 * * * \\
& \mathrm{r}=0.756^{* * *} \\
& \mathrm{r}=0.670^{* * *} \\
& \mathrm{r}=0.669 * * * \\
& \mathrm{r}=0.717 * * *
\end{aligned}
$$

The metals extracted by pyrophosphate, considered to describe the fraction bound by organic matter in soil (McKeAGUE et al. 1971) were, on the average, $38-56 \%$ of the Tamm's oxalate-extractable metals (Table 6). The absolute values of pyrophosphate-extractable metals did not significantly correlate with the organic carbon content in soil. However, there was a low correlation between the organic carbon content and metals expressed as the percentages of Tamm's oxalate-extractable metals. The linear correlation coefficients for the relationship between soil organic carbon content and the percentages of $\mathrm{K}_{4} \mathrm{P}_{2} \mathrm{O}_{7}$-extractable iron and $\mathrm{Na}_{4} \mathrm{P}_{2} \mathrm{O}_{7}$-extractable iron and concentration it appears to precipitate dissolved metals. Ultrafiltration of the centrifugates through $0.025-\mu \mathrm{m}$ filters removes particulate material in a simple and effective way.

In this study, pyrophosphate extracts were cleared by precipitation of suspended clay and organic matter with acid or filtration through $0.2-\mu \mathrm{m}$ filters. The latter method seems to be more certain, as acidification of extracts may dissolve metals from suspended material or, on the other hand, metals may partially remain in precipitates.

$\mathrm{K}_{4} \mathrm{P}_{2} \mathrm{O}_{5}$, used by BAscomв (1968), was used as an extractant because it was thought that 
peptization of soil may be less than if $\mathrm{Na}_{4} \mathrm{P}_{2} \mathrm{O}_{7}$ were used. This opinion was based on the fact that the hydration sphere of $\mathrm{K}^{+}$-ion is smaller than that of $\mathrm{Na}^{+}$-ion. However, lowering the concentration of pyrophosphate from $0.1 \mathrm{M}$ to $0.05 \mathrm{M}$ hardly reduced dispersion of soil. According to Elonen (1971), $0.05 \mathrm{M}$ concentration of $\mathrm{Na}_{4} \mathrm{P}_{2} \mathrm{O}_{7}$ is high enough for peptization of soil in particle-size analysis.

\section{References}

Aleksandrova, L.N. 1960. The use of sodium pyrophosphate for isolating free humic substances and their organic-mineral compounds from the soil. Soviet Soil Sci. 2: 190-197.

BАsсомв, C.L. 1968. Distribution of pyrophosphate-extractable iron and organic carbon in soils of various groups. J. Soil Sci. 19: 251-268.

Elonen, P. 1971. Particle-size analysis of soil. Acta Agr. Fenn. 122: 1-122.

Graham, E.R. 1948. Determination of soil organic matter by means of a photoelectric colorimeter. Soil Sci. 65: 181-183.

McKeague, J.A. 1967. An evaluation of 0.1 M pyrophosphate and pyrophosphate-dithionite in comparison with oxalate as extractants of the accumulation products in Podzols and some other soils. Can. J. Soil Sci. 47: 95-99.

-, Brydon, J.E. \& Miles, N.M. 1971. Differentiation of forms of extractable iron and aluminium in soils. Soil Sci. Soc. Amer. Proc. 35: 33-38.

— \& D^r, J.H. 1966. Dithionite- and oxalate-extractable $\mathrm{Fe}$ and $\mathrm{Al}$ as aids in differentiating various classes of soils. Can. J. Soil Sci. 46: 13-22.

- \& S S chuppli, P.A. 1982. Changes in concentrations of $\mathrm{Fe}$ and $\mathrm{Al}$ in pyrophosphate extracts of soil and composition of sediment resulting from ultracentrifugation in relation to spodic horizon criteria. Soil Sci. 134: $265-270$.

Niskanen, R. 1989. Extractable aluminium, iron and manganese in mineral soils. I Dependence of extractability on the $\mathrm{pH}$ of oxalate, pyrophosphate and EDTA extractants. J. Agric. Sci. Finl. 61: 73-78.

RrTI, R. 1965. On the determination of soil pH. J. Scient. Agric. Soc. Finl. 37: 51-60.

\section{Conclusion}

It seems possible to employ oxalate and pyrophosphate extractants, which are more dilute than conventional reagents. The amounts of metals released by dilute and traditional reagents are closely correlated. In general, extraction methods do not give absolute contents of elements. For example, the amounts extracted increase as the extraction time increases. However, the results obtained with a given method are valuable for soil comparisons.

Schuppli, P.A., Ross, G.J. \& McKeague, J.A. 1983. The effective removal of suspended materials from pyrophosphate extracts of soils from tropical and temperate regions. Soil Sci. Soc. Am. J. 47: 1026-1032.

Schwertmann, U. 1964. Differenzierung der Eisenoxide des Bodens durch photochemische Extraktion mit saurer Ammoniumoxalat-Lösung. Z. Pflanzenernähr. Düng. Bodenk. 105: 194-202.

Searle, P.L. \& Daly, B.K. 1977. The determination of aluminium, iron, manganese and silicon in acid oxalate soil extracts by flame emission and atomic absorption spectrophotometry. Geoderma 19: 1-10.

Sheldrick, B.H. \& McKeague, J.A. 1975. A comparison of extractable $\mathrm{Fe}$ and $\mathrm{Al}$ data using methods followed in the U.S.A. and Canada. Can. J. Soil Sci. 55: $77-78$.

Simmons, W.J. \& Plues-Foster, L.A. 1977. Improved method of analysing difficult soil extracts by flame atomic absorption spectrometry - application to measurement of copper in ammonium oxalate extracts. Aust. J. Soil Res. 15: 171-175.

Tамм, O. 1922: Eine Methode zur Bestimmung der anorganischen Komponente des Gelkomplexes im Boden. Statens Skogsförsöksanstalt, Medd. 19: 387-404.

Webrer, M.D., McKeague, J.A., Raad, A.T., DeKımpe, C.R., Wang, C., Haluschak, P., Stonehouse, H.B., Pettapiece, W.W., Osborne, V.E. \& Green, A.J. 1974. A comparison among nine Canadian laboratories of dithionite-, oxalate-, and pyrophosphate-extractable $\mathrm{Fe}$ and $\mathrm{Al}$ in soils. Can. J. Soil Sci. 54: 293-298.

Ms received January 12, 1988 


\section{Kivennäismaiden uuttuva alumiini, rauta ja mangaani}

II Uuttuvuus oksalaatti- ja pyrofosfaattiliuoksilla

\section{Raina Niskanen}

Helsingin yliopisto, Maanviljelyskemian laitos, 00710 Helsinki

Koska oksalaatti- ja pyrofosfaattiuutteiden analysoinnissa esiintyy vaikeuksia, tutkittiin tavanomaista laimeampien uuttoliuosten käyttöä. Kivennäismaiden alumiinia, rautaa ja mangaania uutettiin $0,05 \mathrm{M}$ oksalaatti- ja pyrofosfaattiliuoksilla sekä vertailuliuoksilla: Tammin oksalaatilla ja $0,1 \mathrm{M}$ natriumpyrofosfaatilla. Tammin oksalaatilla uuttui alumiinia, rautaa ja mangaania keskimäärin 67,81 ja 1,5 mmol/kg maata. Keskimääräinen alumiinin, raudan ja mangaanin uuttuvuus $0,05 \mathrm{M}$ oksalaatilla pH 2,9 ja 4,2 oli 103, 113 ja 87 sekä 72,82 ja
$83 \%$ Tammin oksalaatilla uuttuvasta. Kunkin metallin uuttuvuudet Tammin oksalaatilla ja $0,05 \mathrm{M}$ oksalaateilla korreloivat $r: n$ arvojen vaihdellessa $0,967^{* * *}$ :stä $0,997^{* * *}$ :aaăn $(\mathrm{n}=23)$. Alumiinia, rautaa ja mangaania uuttui $0,1 \mathrm{M}$ natriumpyrofosfaatilla ja $0,05 \mathrm{M}$ kaliumpyrofosfaatilla keskimäärin 38,28 ja 0,6 sekä 33,29 ja $0,6 \mathrm{mmol} / \mathrm{kg}$ maata. Kunkin metallin uuttuvuudet năillă liuoksilla korreloivat $r: n$ arvojen vaihdellessa $0,87^{* * *}$ :stä $0,97^{* * *}$ :aaăn. 